\title{
Optimization of cutting conditions using artificial neural networks and the Edgeworth-Pareto method for CNC face-milling operations on high-strength grade-H steel
}

\author{
Adel Taha Abbas ${ }^{1}$ (D) Danil Yurievich Pimenov ${ }^{2}$ (D) - Ivan Nikolaevich Erdakov ${ }^{3}$ (D) Tadeusz Mikolajczyk $^{4}$ (D) \\ Mahmoud Sayed Soliman ${ }^{1} \cdot$ Magdy Mostafa El Rayes $^{1}$
}

Received: 19 June 2019 / Accepted: 20 August 2019/Published online: 19 October 2019

(C) The Author(s) 2019

\begin{abstract}
Computer Numerical Control (CNC) face milling is commonly used to manufacture products from high-strength grade-H steel in both the automotive and the construction industry. The various milling operations for these components have key performance indicators: accuracy, surface roughness $(R a)$, and machining time for removal of a unit volume $\mathrm{min} / \mathrm{cm}^{3}\left(T_{m}\right)$. The specified surface roughness values for machining each component is achieved based on the prototype specifications. However, poor adherence to specifications can result in the rejection of the machined parts, implying extra production costs and raw material wastage. An algorithm using an artificial neural network (ANN) with the Edgeworth-Pareto method is presented in this paper to optimize the cutting parameter in CNC face-milling operations. The set of parameters are adjusted to improve surface roughness and minimal unit-volume material removal rates, thereby reducing production costs and improving accuracy. An ANN algorithm is designed in Matlab, based on a 3-10-1 Multi-Layer Perceptron (MLP), which predicts the Ra of the workpiece surface to an accuracy of $\pm 5.78 \%$ within the range of the experimental angular spindle speed, feed rate, and cutting depth. An unprecedented Pareto frontier for $R a$ and $T_{m}$ was obtained for the finished grade-H steel workpiece using an ANN algorithm that was then used to determine optimized cutting conditions. Depending on the production objective, one or the other of two sets of optimum machining conditions can be used: the first one sets a minimum cutting power, while the other sets a maximum $T_{m}$ with a slight increase (under 5\%) in milling costs.
\end{abstract}

Keywords Artificial neural network (ANN) CNC O Optimization of cutting parameters · Face milling · Surface roughness · High-strength grade-H steel $\cdot$ Data mining

\section{Introduction}

Nowadays, face milling is widely used in many industries such as machine and machine tool building, the automobile

Danil Yurievich Pimenov

danil_u@ rambler.ru

Tadeusz Mikolajczyk

tami@utp.edu.pl

Adel Taha Abbas

aabbas@ksu.edu.sa

Ivan Nikolaevich Erdakov

wissenschaftler@bk.ru

Mahmoud Sayed Soliman

solimanm@ksu.edu.sa industry, etc. [1]. One of the major control parameters in face milling is surface roughness [2-7]. Grade-H steel materials have various uses and many industrial applications, such as cold-formed components in the automobile industry, among

Magdy Mostafa El Rayes melrayes@ksu.edu.sa

1 Department of Mechanical Engineering, College of Engineering, King Saud University, Riyadh 11421, Kingdom of Saudi Arabia

2 Department of Automated Mechanical Engineering, South Ural State University, Lenin Prosp. 76, Chelyabinsk, Russia 454080

3 Foundry Department, South Ural State University, Lenin Prosp. 76, Chelyabinsk, Russia 454080

4 Department of Production Engineering, UTP University of Science and Technology, Al. prof. S. Kaliskiego 7,

85-796 Bydgoszcz, Poland 
others, on account of their high tensile strength. It is specifically used in safety-related vehicle components, because its high strength means cost-efficient weight reduction of products, without affecting the integrity of the designed components. Increased rigidity of mechanical components can be achieved by using high-strength grade-H steel with no need for further reinforcements. Seat chassis, bumper reinforcement, and door impact beams are typical examples of its use in the automobile industry. Industrial and construction applications of high-strength grade-H steel include tubes for structural applications and hydraulic cylinders and heat- and wearresistant sheet plates [8]. Some examples of precision face milling of high-pressure products from grade-H steel are breech rings and breech blocks for the manufacturing of basic parts of heavy cannons. The limited resources of the modern world mean that maximum efficiency in their use is an overarching objective. The development of resource-saving technologies including those for face milling is therefore becoming crucial. Optimal employment of resources is an important task when machining costly essential components from grade$\mathrm{H}$ steel, as well as surface quality. The minimum machining time per unit volume and the minimum surface roughness, $R a$, are both simultaneously essential. With the fast advance in technological and computational fields, analyzing large bodies of data using AI becomes increasingly relevant.

Research work studying regression models and changes of surface roughness in face milling has been reported in the literature [9-14]. Bruni et al. [9] studied the effect of lubrication-cooling condition and various cutting speeds on surface roughness in the face milling of AISI $420 \mathrm{~B}$. Lela et al. [10] investigated the changes in surface roughness in face milling, corresponding to the changes in cutting speed, feed rate, and depth of cut of St 52-3 steel. They applied three different modeling methodologies to experimental data: Bayesian neural network, regression analysis, and support vector machines. Kovac et al. [11] performed the same study as Lela et al. on AISI 1060 steel. The paper demonstrated the advantage of empirical models using the fuzzy logic modeling technique over traditional regression analysis. Simunovic et al. [12] presented research on machined surface roughness in the face milling of aluminum alloys at various machining parameters. Pimenov [13] presented a geometric model of micro-roughness height in face milling on a machined flat surface, taking into consideration the wear of the cutting tool. Werda et al. [14] performed a similar study on X100CrMoV5 to investigate tool life and surface roughness under different cutting conditions: dry machining; minimum quantity lubrication, through inner channels oriented towards the insert rake face; and minimum quality lubrication through inner channels oriented towards the insert flank face.

Other research work studied roughness prediction models for face-milling applications [15-24]. Baek et al. [15] developed a newly developed model for surface roughness prediction in the face milling of AISI 1045 steel, taking into account both static and dynamic components of the cutting process. Benardos and Vosniakos [16] presented a neural network modeling approach for predicting surface roughness $(R a)$ in the CNC face milling of a series 2 aluminum alloy. Yazdi and Chavoshi [17] studied the influence of cutting parameters and machining force on material removal rate and surface roughness on AL6061 in CNC face-milling operations. The study used two different modeling techniques and showed that the MLP neural network was more powerful than regression analysis, and they simultaneously performed the estimation of $R a$ and MRR. Rosales et al. [18] offered a method for estimating surface roughness depending on spindle speed, feed, cutting depth, tool geometry, and run-out, starting from the register of cutting forces in the process. Bajić et al. [19] investigated the effect of various cutting conditions on surface roughness, tool wear, and cutting forces in face milling for the implementation of off-line process control. Two modeling methodologies, namely regression analysis and neural networks, were used in the study. Chavoshi [20] suggested a model for predicting surface roughness depending on various cutting speeds, feed rates, and cutting depth options in the CNC face milling of Stellite 6 alloy and various softcomputing techniques including multi-layer perceptron (MLP), generalized feed forward (GFF), modular neural network (MNN), and a co-active neuro-fuzzy inference system (CANFIS). Saric et al. [21] modeled surface roughness in the face milling of structural steel S235JRG2. Samtas [22] established surface roughness values in relation to cutting parameters in the face milling of an AISI 1040 steel and aluminum alloy 5083 using optical tools. A program was developed from the study to predict optical surface roughness values using a MATLAB m-file and GUI programming. Sheth and George [23] proposed a model for predicting surface roughness and flatness at different spindle speeds, feed rates, and cutting depths in the face milling of grade B Wrought Cast Steel (WCB). Simunovic et al. [24] offered an analysis on surface roughness in the face milling of Al6060 aluminum alloy based on digital imaging technology of the surfaces that were machined with various spindle speeds, feeds, and cutting depths. Other studies [15-24] proposed models for predicting surface roughness, with no successful optimizations of cutting conditions for better surface roughness.

The following studies may be found on the optimal surface roughness parameters in turning operations [25-30]. Bajić et al. [25] studied the influence of various cutting parameters on surface roughness in the face milling of $\mathrm{St}$ 52-3 carbon steel. The surface roughness prediction models were obtained using two different modeling approaches: regression analysis and neural networks. The optimal cutting parameters were established using the simplex optimization algorithm. Aykut et al. [26] proposed a hybrid NN with genetic algorithm (GONNS) for the 
optimization of cutting conditions in the face milling of workpieces from Stellite 6 with 44 Rockwell C hardness, based on experimental data with minimal operator involvement. End surface roughness and cutting force were measured for different cutting speeds, feed rates, and cutting depths without using coolant. Sukumar et al. [27] used the Taguchi method to identify the optimal combination of influential factors in the milling of an AL 6061 alloy. The input parameters taken into account included speed, feed rate, and depth of cut, and the output parameter was surface roughness. Simunovic et al. [28] investigated the influence of optimal machining parameters on the surface roughness of face-milled structural steel. Moghaddam [29] conducted an experimental and numerical study of the face milling of AISI1045 workpieces. He studied the influence of cutting speed, feed rate, and cutting depths on surface quality. In the last section of his research, a mathematical model was developed for surface roughness prediction using particle swarm optimization (PSO) on the basis of experimental results. Rodríguez et al. [30] used artificial intelligence to optimize choosing the right cutting tools in the design of face-milling operations, where end surface roughness is a key criterion. However, the solutions presented in studies [25-30] for establishing the optimum cutting conditions only took account of final surface quality, ignoring its relation to machining performance and machining time per unit volume, which is unacceptable when machining such expensive materials as grade- $\mathrm{H}$ steel.

Considering the above, let us now look at the studies that establish the optimal parameters of face milling using multi-objective optimization [31-33]. Fratila and Caizar [31] outlined the Taguchi optimization methodology, which is applied to optimize the cutting parameters in face milling when machining $\mathrm{AlMg}_{3}$ with a high-speed steel (HSS) tool, in order to obtain the best surface roughness with minimum power consumption. Yang et al. [32] solved the problem of multi-objective optimization of multi-pass face-milling parameters using Pareto optimal solutions. The optimization consists in simultaneously minimizing production time and cost and maximizing profit rate, conditional upon satisfying the constraints on machine power, cutting force, machining speed, feed rate, and surface roughness. Abbas et al. [33] investigated the effect of changing cutting parameters using a full-factorial technique; the studied parameters were the speed $(n)$, depth of cut $\left(a_{p}\right)$, and feed $\left(v_{f}\right)$, and the measured parameters were the surface roughness parameters $\left(R a\right.$ and $\left.R_{t}\right)$. Multiobjective optimization was used for minimizing $R a$ and for maximizing the metal removal rate, $Q$, and then the results were presented. There are therefore few papers discussing multi-objective optimization of face milling that have stated that the most prominent method to solve this kind of problems is considered to be the Pareto method.
However, in studies [31-33], no multi-objective optimization of grade-H steel machining is featured. This kind of material is widely used when materials have to be of high operational strength. Considering the high cost of this material, the required surface roughness has to be precisely set and the unit-volume machining time has to be minimized.

The objective of this paper is therefore to establish the facemilling conditions of grade $\mathrm{H}$ steel that provide for either minimum cutting power or maximum machining time per unit volume, $T_{m}$, while maintaining the design $R a$, and taking into account the cost of machining based on an ANN model for predicting surface roughness.

\section{Materials and method}

\subsection{Experimental conditions}

Grade-H high-strength steel is used for testing specimens. Table 1 shows its chemical composition following the DEFSTAN 10-13/2005 standard. The heat treatment consisted of heating samples to $870{ }^{\circ} \mathrm{C}$ for $4 \mathrm{~h}$ followed by oil quenching. Subsequently, heat was applied in a tempering process for $1.5 \mathrm{~h}$ at $650{ }^{\circ} \mathrm{C}$ and was maintained over a $10-\mathrm{h}$ period, followed by air cooling. Table 2 shows the mechanical properties.

The machining of the test specimens was performed on a vertical mill as shown in Fig. 1. The surface area of the sample had the following dimensions $b=40 \mathrm{~mm}, l=$ $100 \mathrm{~mm}$, and $h=60 \mathrm{~mm}$. The width of cut for all the runs was $40 \mathrm{~mm}$. The cutting tools with carbide inserts were used in a face mill with a Sandvik R245-063Q22-12M tool holder and Sandvik carbide-coated inserts R245-12 T3MPM4240. The cutter diameter was $d=63 \mathrm{~mm}$ with $z=5$ edges. This tool is known for providing high-quality surface finishes with efficient material removal rates. It is commonly used for all types of materials from stainless steel to titanium alloys. The investigation was divided into 25 groups of five runs. Clusters of five groups were subject to one common spindle speed $n$ starting from $400 \mathrm{rpm}$ with a 100 increment till $800 \mathrm{rpm}$. Depth of cut $a_{p}$ varied (0.50, $0.75,1.00,1.25,1.50 \mathrm{~mm})$. Feed rate, $v_{f}$, varied $(50,75$, $100,125,150 \mathrm{~mm} / \mathrm{min})$. A TESA Rugosurf $90-\mathrm{G}$ model was used for surface roughness measurement.

Table 1 Chemical composition for $\mathrm{H}$-steel material according to DEFSTAN 10-13/2005

\begin{tabular}{lllllllll}
\hline $\mathrm{C}$ & $\mathrm{Cr}$ & $\mathrm{Mn}$ & $\mathrm{Mo}$ & $\mathrm{Ni}$ & $\mathrm{P}$ & $\mathrm{S}$ & $\mathrm{Si}$ & $\mathrm{V}$ \\
\hline 0.30 & 0.76 & 0.46 & 0.47 & 2.86 & 0.006 & 0.002 & 0.17 & 0.01 \\
0.36 & 0.94 & 0.53 & 0.58 & 3.10 & 0.011 & 0.01 & 0.32 & 0.03 \\
\hline
\end{tabular}


Table 2 Mechanical properties for $\mathrm{H}$-steel according to DEFSTAN 10-13/2005

\begin{tabular}{lllllll}
\hline $\begin{array}{l}\text { Mechanical } \\
\text { properties }\end{array}$ & $\begin{array}{l}\text { Ultimate tensile } \\
\text { strength }\end{array}$ & $\begin{array}{l}0.2 \% \text { Proof } \\
\text { Strength }\end{array}$ & $\begin{array}{l}\text { Elongation } \\
\%\end{array}$ & $\begin{array}{l}\text { Reduction } \\
\text { of area }\end{array}$ & $\begin{array}{l}\text { Charpy } \\
\text { at } \\
(-\end{array}$ & Hardness \\
& & & & $400 \mathrm{C})$ & \\
\hline Value & $>1200 \mathrm{n} / \mathrm{mm}^{2}$ & $\begin{array}{c}850-1000 \\
\mathrm{n} / \mathrm{mm}^{2}\end{array}$ & $\geq 10 \%$ & $>27 \%$ & $\geq 24.4 \mathrm{~J}$ & $\begin{array}{r}32-38 \\
\text { HRC }\end{array}$ \\
\hline
\end{tabular}

\subsection{Strategy for determining the optimal conditions}

ANN milling model based on experimental data [34-36] was built to achieve our objective of solving the optimization problem in a multi-objective setting using both Edgeworth and the Pareto frontiers [31-33, 37-39].

The strategy for determining the optimum conditions was as follows:

Step 1. Define both the limitations and the boundary conditions to set the optimization criteria. Define the working domain.

Step 2. Perform an approximation of three variable functions using Data Mining technique [40-43], based on experimental data and $\mathrm{NN}$.

Step 3. Identify the Pareto curve: optimal decisions and estimates.

Step 4. Determine Pareto non-dominated estimates.

Step 5. Establish a set of Pareto points that may contain an optimum decision.

As shown in the diagram in Fig. 2, the experimental machining parameter settings were agreed upon between the researchers and the experts. The experts then went on to develop the set of optimal decisions, and the decisionmaker selected the most appropriate one. The symbols in use were the same ones used in [44]: DM-decision; $F Z$-valid estimates; $f=\left(f_{1}, f_{2}, \ldots, \mathrm{f}_{\mathrm{m}}\right)$-objective function; $Y=\mathrm{f}(X)$ - the set of estimates; Ndom $X$ - set of non-dominated estimates; Ndom $Y$-set of non-dominated estimates; $P_{f}(X)$ - the set of Pareto optimal frontiers; $P$ (set of Pareto optimal vectors-Pareto optimal estimates).

\section{Results and discussion}

The first three steps followed in the research are described below.

\subsection{Optimization problem statement (the first step of the strategy)}

Based on the objective, the optimization criteria in the milling of the cubic workpiece were established as $f_{l}$ surface roughness $(R a, \mu \mathrm{m})$ and $f_{2}$ machining time per unit volume $\left(T_{m}, \mathrm{~min} / \mathrm{cm}^{3}\right)$, i.e., $m=2$. Consequently, a set of possible $Y$ estimates in the two-dimensional space, $R^{2}$, formed the vectors $f=\left(f_{1}, f_{2}\right)$. A search was then performed for a set of estimates that would have the minimum $f$ vector lengths. The criteria were normalized and placed in a dimensionless form.

Table 3, 4, 5, 6, and 7 shows the results of experiments and also includes the parameters and limitations to the optimization problem: $x_{1}=[400 \div 800]-$ spindle speed, $n$, rpm; $x_{2}=[0.5 \div 1.5]$-depth of cut, $a_{p}, \mathrm{~mm} ; x_{3}=[50 \div 150]$-feed rate, $v_{f}, \mathrm{~mm} / \mathrm{min}$.

Fig. 1 Machined workpiece setup

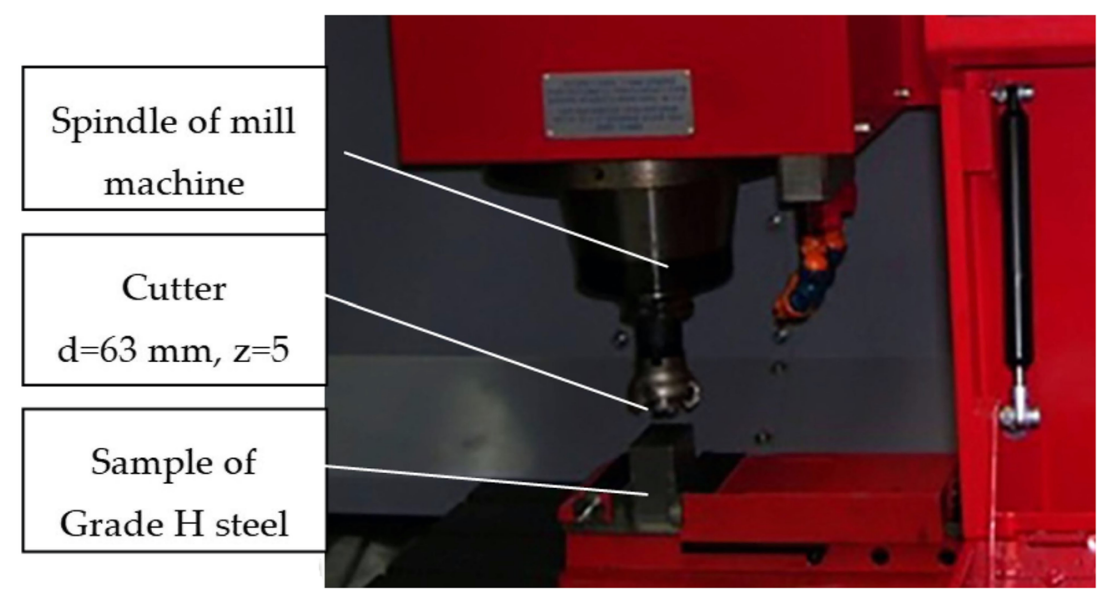


Fig. 2 Flow chart for the multiobjective optimization problem

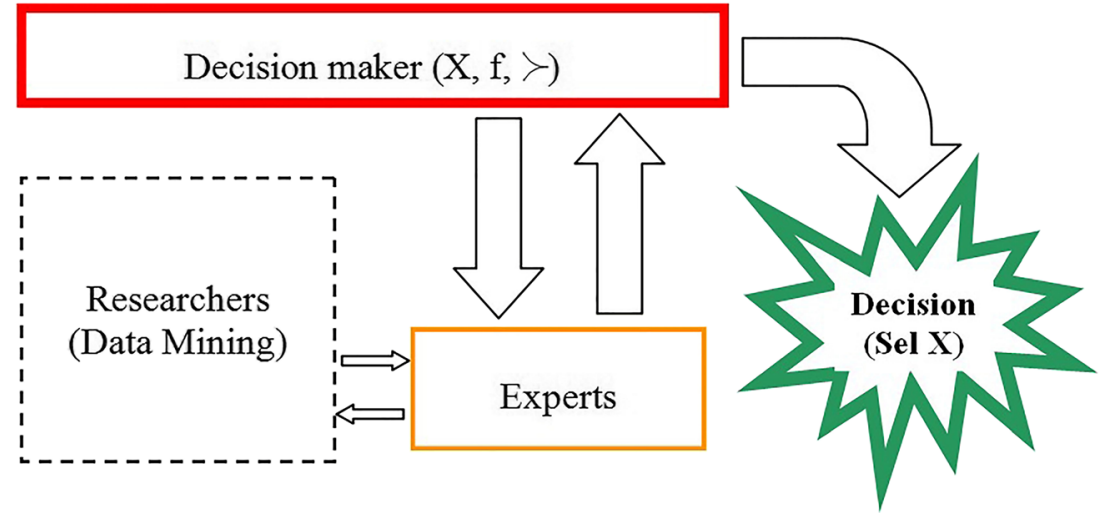

Surface roughness $(R a, \mu \mathrm{m})$ was measured, and the machining time was calculated as follows:

$T_{m}=1000 /\left(v_{c} \times a_{p} \times v_{f}\right)$
The dimensionless surface roughness value, $f_{1}\left(R a^{*}\right)$, and the machining time per unit volume, $f_{2}\left(T_{m}{ }^{*}\right)$, were calculated on the basis of the following formulas:
Table 3 Results of experiment and optimization parameter values for variable workpiece milling at $a_{p}=0.5 \mathrm{~mm}$

\begin{tabular}{|c|c|c|c|c|c|c|c|}
\hline \multicolumn{3}{|c|}{ Variables } & \multicolumn{5}{|c|}{ Optimization parameters } \\
\hline & & & \multicolumn{2}{|c|}{ Results of experiment } & \multicolumn{3}{|c|}{ Dimensionless parameters } \\
\hline $\begin{array}{l}x_{1} \\
\text { spindle } \\
\text { speed, } \\
n, \\
(\mathrm{rpm})\end{array}$ & $\begin{array}{l}\mathrm{x}_{2} \\
\text { depth } \\
\text { of cut, } \\
a_{p}, \\
(\mathrm{~mm})\end{array}$ & $\begin{array}{l}x_{3} \text { feed } \\
\text { rate, } \\
v_{f}, \\
(\mathrm{~mm} / \\
\mathrm{min})\end{array}$ & $\begin{array}{l}\text { Surface } \\
\text { roughness, } \\
R a(\mu \mathrm{m})\end{array}$ & $\begin{array}{l}\text { Unit- } \\
\text { volume } \\
\text { machining } \\
\text { time, } T m \\
\left(\mathrm{~min} / \mathrm{cm}^{3}\right)\end{array}$ & $\begin{array}{l}\text { Dimensionless } \\
\text { surface } \\
\text { roughness, } f_{1} \\
\left(R a^{*}\right), \text { unit }\end{array}$ & $\begin{array}{l}\text { Dimensionless } \\
\text { unit-volume } \\
\text { machining } \\
\text { time, } f_{2}\left(T m^{*}\right) \text {, } \\
\text { unit }\end{array}$ & $\begin{array}{l}\text { Unit } \\
\text { vector } \\
\text { length, } f \text {, } \\
\text { estimates }\end{array}$ \\
\hline 400 & 0.5 & 50 & 0.164 & 1 & 0.672 & 1.000 & 1.204 \\
\hline 400 & 0.5 & 75 & 0.175 & 0.667 & 0.717 & 0.667 & 0.979 \\
\hline 400 & 0.5 & 100 & 0.188 & 0.5 & 0.770 & 0.500 & 0.918 \\
\hline 400 & 0.5 & 125 & 0.214 & 0.4 & 0.877 & 0.400 & 0.964 \\
\hline 400 & 0.5 & 150 & 0.218 & 0.333 & 0.893 & 0.333 & 0.953 \\
\hline 500 & 0.5 & 50 & 0.147 & 1 & 0.602 & 1.000 & 1.167 \\
\hline 500 & 0.5 & 75 & 0.155 & 0.667 & 0.635 & 0.667 & 0.921 \\
\hline 500 & 0.5 & 100 & 0.169 & 0.5 & 0.693 & 0.500 & 0.854 \\
\hline 500 & 0.5 & 125 & 0.192 & 0.4 & 0.787 & 0.400 & 0.882 \\
\hline 500 & 0.5 & 150 & 0.196 & 0.333 & 0.803 & 0.333 & 0.869 \\
\hline 600 & 0.5 & 50 & 0.076 & 1 & 0.311 & 1.000 & 1.047 \\
\hline 600 & 0.5 & 75 & 0.085 & 0.667 & 0.348 & 0.667 & 0.752 \\
\hline 600 & 0.5 & 100 & 0.098 & 0.5 & 0.402 & 0.500 & 0.641 \\
\hline 600 & 0.5 & 125 & 0.108 & 0.4 & 0.443 & 0.400 & 0.596 \\
\hline 600 & 0.5 & 150 & 0.115 & 0.333 & 0.471 & 0.333 & 0.577 \\
\hline 700 & 0.5 & 50 & 0.073 & 1 & 0.299 & 1.000 & 1.043 \\
\hline 700 & 0.5 & 75 & 0.08 & 0.667 & 0.328 & 0.667 & 0.743 \\
\hline 700 & 0.5 & 100 & 0.1 & 0.5 & 0.410 & 0.500 & 0.646 \\
\hline 700 & 0.5 & 125 & 0.14 & 0.4 & 0.574 & 0.400 & 0.699 \\
\hline 700 & 0.5 & 150 & 0.122 & 0.333 & 0.500 & 0.333 & 0.600 \\
\hline 800 & 0.5 & 50 & 0.07 & 1 & 0.287 & 1.000 & 1.040 \\
\hline 800 & 0.5 & 75 & 0.076 & 0.667 & 0.311 & 0.667 & 0.736 \\
\hline 800 & 0.5 & 100 & 0.11 & 0.5 & 0.451 & 0.500 & 0.673 \\
\hline 800 & 0.5 & 125 & 0.117 & 0.4 & 0.480 & 0.400 & 0.624 \\
\hline 800 & 0.5 & 150 & 0.135 & 0.333 & 0.553 & 0.333 & 0.645 \\
\hline
\end{tabular}


Table 4 Results of experiment and optimization parameter values for variable workpiece milling at $a_{p}=0.75 \mathrm{~mm}$

\begin{tabular}{|c|c|c|c|c|c|c|c|}
\hline \multicolumn{3}{|c|}{ Variables } & \multicolumn{5}{|c|}{ Optimization parameters } \\
\hline & & & \multicolumn{2}{|c|}{ Results of experiment } & \multicolumn{3}{|c|}{ Dimensionless parameters } \\
\hline $\begin{array}{l}x_{1} \\
\text { spindle } \\
\text { speed, } \\
n, \\
(\mathrm{rpm})\end{array}$ & $\begin{array}{l}\mathrm{x}_{2} \\
\text { depth } \\
\text { of cut, } \\
a_{p} \\
(\mathrm{~mm})\end{array}$ & $\begin{array}{l}x_{3} \text { feed } \\
\text { rate, } \\
v_{f} \\
(\mathrm{~mm} / \\
\mathrm{min})\end{array}$ & $\begin{array}{l}\text { Surface } \\
\text { roughness, } \\
R a(\mu \mathrm{m})\end{array}$ & $\begin{array}{l}\text { Unit- } \\
\text { volume } \\
\text { machining } \\
\text { time, } T m \\
\left(\mathrm{~min} / \mathrm{cm}^{3}\right)\end{array}$ & $\begin{array}{l}\text { Dimensionless } \\
\text { surface } \\
\text { roughness, } f_{1} \\
\left(R a^{*}\right), \text { unit }\end{array}$ & $\begin{array}{l}\text { Dimensionless } \\
\text { unit-volume } \\
\text { machining } \\
\text { time, } f_{2}\left(T m^{*}\right) \text {, } \\
\text { unit }\end{array}$ & $\begin{array}{l}\text { Unit } \\
\text { vector } \\
\text { length, } f \text {, } \\
\text { estimates }\end{array}$ \\
\hline 400 & 0.75 & 50 & 0.119 & 0.667 & 0.488 & 0.667 & 0.826 \\
\hline 400 & 0.75 & 75 & 0.187 & 0.444 & 0.766 & 0.444 & 0.886 \\
\hline 400 & 0.75 & 100 & 0.193 & 0.333 & 0.791 & 0.333 & 0.858 \\
\hline 400 & 0.75 & 125 & 0.199 & 0.267 & 0.816 & 0.267 & 0.858 \\
\hline 400 & 0.75 & 150 & 0.202 & 0.222 & 0.828 & 0.222 & 0.857 \\
\hline 500 & 0.75 & 50 & 0.107 & 0.667 & 0.439 & 0.667 & 0.798 \\
\hline 500 & 0.75 & 75 & 0.168 & 0.444 & 0.689 & 0.444 & 0.819 \\
\hline 500 & 0.75 & 100 & 0.173 & 0.333 & 0.709 & 0.333 & 0.783 \\
\hline 500 & 0.75 & 125 & 0.179 & 0.267 & 0.734 & 0.267 & 0.781 \\
\hline 500 & 0.75 & 150 & 0.188 & 0.222 & 0.770 & 0.222 & 0.802 \\
\hline 600 & 0.75 & 50 & 0.063 & 0.667 & 0.258 & 0.667 & 0.715 \\
\hline 600 & 0.75 & 75 & 0.075 & 0.444 & 0.307 & 0.444 & 0.540 \\
\hline 600 & 0.75 & 100 & 0.097 & 0.333 & 0.398 & 0.333 & 0.519 \\
\hline 600 & 0.75 & 125 & 0.105 & 0.267 & 0.430 & 0.267 & 0.506 \\
\hline 600 & 0.75 & 150 & 0.124 & 0.222 & 0.508 & 0.222 & 0.555 \\
\hline 700 & 0.75 & 50 & 0.085 & 0.667 & 0.348 & 0.667 & 0.752 \\
\hline 700 & 0.75 & 75 & 0.098 & 0.444 & 0.402 & 0.444 & 0.599 \\
\hline 700 & 0.75 & 100 & 0.121 & 0.333 & 0.496 & 0.333 & 0.597 \\
\hline 700 & 0.75 & 125 & 0.134 & 0.267 & 0.549 & 0.267 & 0.611 \\
\hline 700 & 0.75 & 150 & 0.147 & 0.222 & 0.602 & 0.222 & 0.642 \\
\hline 800 & 0.75 & 50 & 0.114 & 0.667 & 0.467 & 0.667 & 0.814 \\
\hline 800 & 0.75 & 75 & 0.12 & 0.444 & 0.492 & 0.444 & 0.663 \\
\hline 800 & 0.75 & 100 & 0.132 & 0.333 & 0.541 & 0.333 & 0.635 \\
\hline 800 & 0.75 & 125 & 0.166 & 0.267 & 0.680 & 0.267 & 0.731 \\
\hline 800 & 0.75 & 150 & 0.172 & 0.222 & 0.705 & 0.222 & 0.739 \\
\hline
\end{tabular}

Table 5 Results of experiment and optimization parameter values for variable workpiece milling at $a_{p}=1.0 \mathrm{~mm}$

\begin{tabular}{|c|c|c|c|c|c|c|c|}
\hline \multicolumn{3}{|c|}{ Variables } & \multicolumn{5}{|c|}{ Optimization parameters } \\
\hline & & & \multicolumn{2}{|c|}{ Results of experiment } & \multicolumn{3}{|c|}{ Dimensionless parameters } \\
\hline $\begin{array}{l}x_{1} \\
\text { spindle } \\
\text { speed, } \\
n, \\
\text { (rpm) }\end{array}$ & $\begin{array}{l}\mathrm{x}_{2} \\
\text { depth } \\
\text { of cut, } \\
a_{p}, \\
(\mathrm{~mm})\end{array}$ & $\begin{array}{l}x_{3} \text { feed } \\
\text { rate, } \\
v_{f}, \\
(\mathrm{~mm} / \\
\min )\end{array}$ & $\begin{array}{l}\text { Surface } \\
\text { roughness, } \\
R a(\mu \mathrm{m})\end{array}$ & $\begin{array}{l}\text { Unit- } \\
\text { volume } \\
\text { machining } \\
\text { time, } T m \\
\left(\mathrm{~min} / \mathrm{cm}^{3}\right)\end{array}$ & $\begin{array}{l}\text { Dimensionless } \\
\text { surface } \\
\text { roughness, } f_{1} \\
\left(R a^{*}\right) \text {, unit }\end{array}$ & $\begin{array}{l}\text { Dimensionless } \\
\text { unit-volume } \\
\text { machining } \\
\text { time, } f_{2}\left(T m^{*}\right) \text {, } \\
\text { unit }\end{array}$ & $\begin{array}{l}\text { Unit } \\
\text { vector } \\
\text { length, } f \text {, } \\
\text { estimates }\end{array}$ \\
\hline 400 & 1 & 50 & 0.103 & 0.5 & 0.422 & 0.500 & 0.654 \\
\hline 400 & 1 & 75 & 0.14 & 0.333 & 0.574 & 0.333 & 0.663 \\
\hline 400 & 1 & 100 & 0.155 & 0.25 & 0.635 & 0.250 & 0.683 \\
\hline 400 & 1 & 125 & 0.168 & 0.2 & 0.689 & 0.200 & 0.717 \\
\hline 400 & 1 & 150 & 0.172 & 0.167 & 0.705 & 0.167 & 0.724 \\
\hline 500 & 1 & 50 & 0.092 & 0.5 & 0.377 & 0.500 & 0.626 \\
\hline 500 & 1 & 75 & 0.126 & 0.333 & 0.516 & 0.333 & 0.614 \\
\hline 500 & 1 & 100 & 0.139 & 0.25 & 0.570 & 0.250 & 0.622 \\
\hline 500 & 1 & 125 & 0.151 & 0.2 & 0.619 & 0.200 & 0.650 \\
\hline 500 & 1 & 150 & 0.158 & 0.167 & 0.648 & 0.167 & 0.669 \\
\hline 600 & 1 & 50 & 0.075 & 0.5 & 0.307 & 0.500 & 0.587 \\
\hline 600 & 1 & 75 & 0.082 & 0.333 & 0.336 & 0.333 & 0.473 \\
\hline 600 & 1 & 100 & 0.115 & 0.25 & 0.471 & 0.250 & 0.534 \\
\hline 600 & 1 & 125 & 0.123 & 0.2 & 0.504 & 0.200 & 0.542 \\
\hline 600 & 1 & 150 & 0.148 & 0.167 & 0.607 & 0.167 & 0.629 \\
\hline 700 & 1 & 50 & 0.116 & 0.5 & 0.475 & 0.500 & 0.690 \\
\hline 700 & 1 & 75 & 0.122 & 0.333 & 0.500 & 0.333 & 0.601 \\
\hline 700 & 1 & 100 & 0.145 & 0.25 & 0.594 & 0.250 & 0.645 \\
\hline 700 & 1 & 125 & 0.158 & 0.2 & 0.648 & 0.200 & 0.678 \\
\hline 700 & 1 & 150 & 0.184 & 0.167 & 0.754 & 0.167 & 0.772 \\
\hline 800 & 1 & 50 & 0.129 & 0.5 & 0.529 & 0.500 & 0.728 \\
\hline 800 & 1 & 75 & 0.14 & 0.333 & 0.574 & 0.333 & 0.663 \\
\hline 800 & 1 & 100 & 0.181 & 0.25 & 0.742 & 0.250 & 0.783 \\
\hline 800 & 1 & 125 & 0.196 & 0.2 & 0.803 & 0.200 & 0.828 \\
\hline 800 & 1 & 150 & 0.22 & 0.167 & 0.902 & 0.167 & 0.917 \\
\hline
\end{tabular}


Table 6 Results of experiment and optimization parameter values for variable workpiece milling at $a_{p}=1.25 \mathrm{~mm}$

\begin{tabular}{|c|c|c|c|c|c|c|c|}
\hline \multicolumn{3}{|c|}{ Variables } & \multicolumn{5}{|c|}{ Optimization parameters } \\
\hline & & & \multicolumn{2}{|c|}{ Results of experiment } & \multicolumn{3}{|c|}{ Dimensionless parameters } \\
\hline $\begin{array}{l}x_{1} \\
\text { spindle } \\
\text { speed, } \\
n, \\
(\mathrm{rpm})\end{array}$ & $\begin{array}{l}\mathrm{x}_{2} \\
\text { depth } \\
\text { of cut, } \\
a_{p} \\
(\mathrm{~mm})\end{array}$ & $\begin{array}{l}x_{3} \text { feed } \\
\text { rate, } \\
v_{f} \\
(\mathrm{~mm} / \\
\mathrm{min})\end{array}$ & $\begin{array}{l}\text { Surface } \\
\text { roughness, } \\
R a(\mu \mathrm{m})\end{array}$ & $\begin{array}{l}\text { Unit- } \\
\text { volume } \\
\text { machining } \\
\text { time, } T m \\
\left(\mathrm{~min} / \mathrm{cm}^{3}\right)\end{array}$ & $\begin{array}{l}\text { Dimensionless } \\
\text { surface } \\
\text { roughness, } f_{1} \\
\left(R a^{*}\right), \text { unit }\end{array}$ & $\begin{array}{l}\text { Dimensionless } \\
\text { unit-volume } \\
\text { machining } \\
\text { time, } f_{2}\left(T m^{*}\right) \text {, } \\
\text { unit }\end{array}$ & $\begin{array}{l}\text { Unit } \\
\text { vector } \\
\text { length, } f \text {, } \\
\text { estimates }\end{array}$ \\
\hline 400 & 1.25 & 50 & 0.144 & 0.4 & 0.590 & 0.400 & 0.713 \\
\hline 400 & 1.25 & 75 & 0.157 & 0.267 & 0.643 & 0.267 & 0.697 \\
\hline 400 & 1.25 & 100 & 0.164 & 0.2 & 0.672 & 0.200 & 0.701 \\
\hline 400 & 1.25 & 125 & 0.185 & 0.16 & 0.758 & 0.160 & 0.775 \\
\hline 400 & 1.25 & 150 & 0.198 & 0.133 & 0.811 & 0.133 & 0.822 \\
\hline 500 & 1.25 & 50 & 0.129 & 0.4 & 0.529 & 0.400 & 0.663 \\
\hline 500 & 1.25 & 75 & 0.141 & 0.267 & 0.578 & 0.267 & 0.637 \\
\hline 500 & 1.25 & 100 & 0.147 & 0.2 & 0.602 & 0.200 & 0.635 \\
\hline 500 & 1.25 & 125 & 0.166 & 0.16 & 0.680 & 0.160 & 0.699 \\
\hline 500 & 1.25 & 150 & 0.178 & 0.133 & 0.730 & 0.133 & 0.742 \\
\hline 600 & 1.25 & 50 & 0.126 & 0.4 & 0.516 & 0.400 & 0.653 \\
\hline 600 & 1.25 & 75 & 0.132 & 0.267 & 0.541 & 0.267 & 0.603 \\
\hline 600 & 1.25 & 100 & 0.137 & 0.2 & 0.561 & 0.200 & 0.596 \\
\hline 600 & 1.25 & 125 & 0.14 & 0.16 & 0.574 & 0.160 & 0.596 \\
\hline 600 & 1.25 & 150 & 0.145 & 0.133 & 0.594 & 0.133 & 0.609 \\
\hline 700 & 1.25 & 50 & 0.129 & 0.4 & 0.529 & 0.400 & 0.663 \\
\hline 700 & 1.25 & 75 & 0.139 & 0.267 & 0.570 & 0.267 & 0.629 \\
\hline 700 & 1.25 & 100 & 0.157 & 0.2 & 0.643 & 0.200 & 0.674 \\
\hline 700 & 1.25 & 125 & 0.179 & 0.16 & 0.734 & 0.160 & 0.751 \\
\hline 700 & 1.25 & 150 & 0.187 & 0.133 & 0.766 & 0.133 & 0.778 \\
\hline 800 & 1.25 & 50 & 0.134 & 0.4 & 0.549 & 0.400 & 0.679 \\
\hline 800 & 1.25 & 75 & 0.145 & 0.267 & 0.594 & 0.267 & 0.651 \\
\hline 800 & 1.25 & 100 & 0.188 & 0.2 & 0.770 & 0.200 & 0.796 \\
\hline 800 & 1.25 & 125 & 0.203 & 0.16 & 0.832 & 0.160 & 0.847 \\
\hline 800 & 1.25 & 150 & 0.228 & 0.133 & 0.934 & 0.133 & 0.944 \\
\hline
\end{tabular}

$T_{m}{ }^{*}=T_{m i} / T_{m \max }$

\subsection{Creating an ANN-based surface roughness prediction model (the second step of the strategy)}

A licensed version of Matlab R2010b served as a tool for building the neural network employed in this paper. The Levenberg-Marquardt algorithm was employed to train the NN. Two layers were used to structure the NN: a layer of sigmoid neurons and a linear layer of output neurons. A normalization process was used for all values. It ensured that all inputs complied with the $\{0,1\}$ domain to enhance the NN training process. The overfitting was dealt with by enhancing the generalization process of the network. A training set was used to update the weighted values of the neurons and a validation set for stopping the training in case of errors. The least mean squared error was used to establish the number of neurons in the hidden layer. 
Table 7 Results of experiment and optimization parameter values for variable workpiece milling at $a_{p}=1.5 \mathrm{~mm}$

\begin{tabular}{|c|c|c|c|c|c|c|c|}
\hline \multirow{2}{*}{\multicolumn{3}{|c|}{ Variables }} & \multicolumn{5}{|c|}{ Optimization parameters } \\
\hline & & & \multicolumn{2}{|c|}{ Results of experiment } & \multicolumn{3}{|c|}{ Dimensionless parameters } \\
\hline $\begin{array}{l}x_{1} \\
\text { spindle } \\
\text { speed, } \\
n, \\
(\mathrm{rpm})\end{array}$ & $\begin{array}{l}\mathrm{x}_{2} \\
\text { depth } \\
\text { of cut, } \\
a_{p} \\
(\mathrm{~mm})\end{array}$ & $\begin{array}{l}x_{3} \text { feed } \\
\text { rate, } \\
v_{f} \\
(\mathrm{~mm} / \\
\mathrm{min})\end{array}$ & $\begin{array}{l}\text { Surface } \\
\text { roughness, } \\
R a(\mu \mathrm{m})\end{array}$ & $\begin{array}{l}\text { Unit- } \\
\text { volume } \\
\text { machining } \\
\text { time, } T m \\
\left(\mathrm{~min} / \mathrm{cm}^{3}\right)\end{array}$ & $\begin{array}{l}\text { Dimensionless } \\
\text { surface } \\
\text { roughness, } f_{1} \\
\left(R a^{*}\right), \text { unit }\end{array}$ & $\begin{array}{l}\text { Dimensionless } \\
\text { unit-volume } \\
\text { machining } \\
\text { time, } f_{2}\left(T m^{*}\right) \text {, } \\
\text { unit }\end{array}$ & $\begin{array}{l}\text { Unit } \\
\text { vector } \\
\text { length, } f \text {, } \\
\text { estimates }\end{array}$ \\
\hline 400 & 1.5 & 50 & 0.104 & 0.333 & 0.426 & 0.333 & 0.541 \\
\hline 400 & 1.5 & 75 & 0.192 & 0.222 & 0.787 & 0.222 & 0.818 \\
\hline 400 & 1.5 & 100 & 0.202 & 0.167 & 0.828 & 0.167 & 0.845 \\
\hline 400 & 1.5 & 125 & 0.208 & 0.133 & 0.852 & 0.133 & 0.863 \\
\hline 400 & 1.5 & 150 & 0.215 & 0.111 & 0.881 & 0.111 & 0.888 \\
\hline 500 & 1.5 & 50 & 0.093 & 0.333 & 0.381 & 0.333 & 0.506 \\
\hline 500 & 1.5 & 75 & 0.172 & 0.222 & 0.705 & 0.222 & 0.739 \\
\hline 500 & 1.5 & 100 & 0.181 & 0.167 & 0.742 & 0.167 & 0.760 \\
\hline 500 & 1.5 & 125 & 0.187 & 0.133 & 0.766 & 0.133 & 0.778 \\
\hline 500 & 1.5 & 150 & 0.195 & 0.111 & 0.799 & 0.111 & 0.807 \\
\hline 600 & 1.5 & 50 & 0.088 & 0.333 & 0.361 & 0.333 & 0.491 \\
\hline 600 & 1.5 & 75 & 0.11 & 0.222 & 0.451 & 0.222 & 0.503 \\
\hline 600 & 1.5 & 100 & 0.119 & 0.167 & 0.488 & 0.167 & 0.516 \\
\hline 600 & 1.5 & 125 & 0.123 & 0.133 & 0.504 & 0.133 & 0.521 \\
\hline 600 & 1.5 & 150 & 0.126 & 0.111 & 0.516 & 0.111 & 0.528 \\
\hline 700 & 1.5 & 50 & 0.126 & 0.333 & 0.516 & 0.333 & 0.614 \\
\hline 700 & 1.5 & 75 & 0.141 & 0.222 & 0.578 & 0.222 & 0.619 \\
\hline 700 & 1.5 & 100 & 0.167 & 0.167 & 0.684 & 0.167 & 0.705 \\
\hline 700 & 1.5 & 125 & 0.185 & 0.133 & 0.758 & 0.133 & 0.770 \\
\hline 700 & 1.5 & 150 & 0.196 & 0.111 & 0.803 & 0.111 & 0.811 \\
\hline 800 & 1.5 & 50 & 0.142 & 0.333 & 0.582 & 0.333 & 0.671 \\
\hline 800 & 1.5 & 75 & 0.155 & 0.222 & 0.635 & 0.222 & 0.673 \\
\hline 800 & 1.5 & 100 & 0.197 & 0.167 & 0.807 & 0.167 & 0.824 \\
\hline 800 & 1.5 & 125 & 0.215 & 0.133 & 0.881 & 0.133 & 0.891 \\
\hline 800 & 1.5 & 150 & 0.244 & 0.111 & 1.000 & 0.111 & 1.006 \\
\hline
\end{tabular}

First of all, the multi-layer perceptrons were trained with 9-11 neurons in the hidden layer, with $15 \%$ of the records used to validate the model. The lowest error values are presented in Fig. 3.
An analysis of the graphical functions in Fig. $3 \mathrm{a}-\mathrm{c}$ led to the conclusion that the lowest error of $0.23 \%$ in the validation set was provided by MLP 3-10-1. The determination coefficient of the model was 0.942 , which reflects
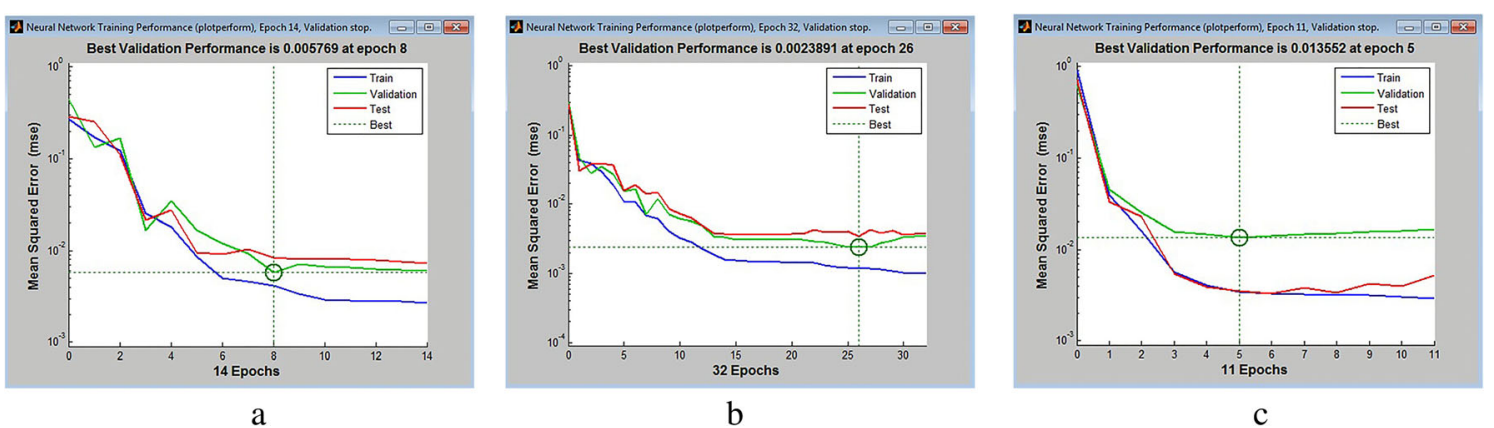

Fig. 3 The lowest mean squared error for the validation set in the ... configuration (calculated in Matlab). a MLP 3-9-1. b MLP 3-10-1. c MLP 3-11-1 
Fig. 4 The lowest mean squared error in generalizing the experimental data in MLP 3-10-1 (a) with various validation sets: $\mathbf{b}$ $10 \%$ and $\mathbf{c} 20 \%$ (calculated in Matlab)
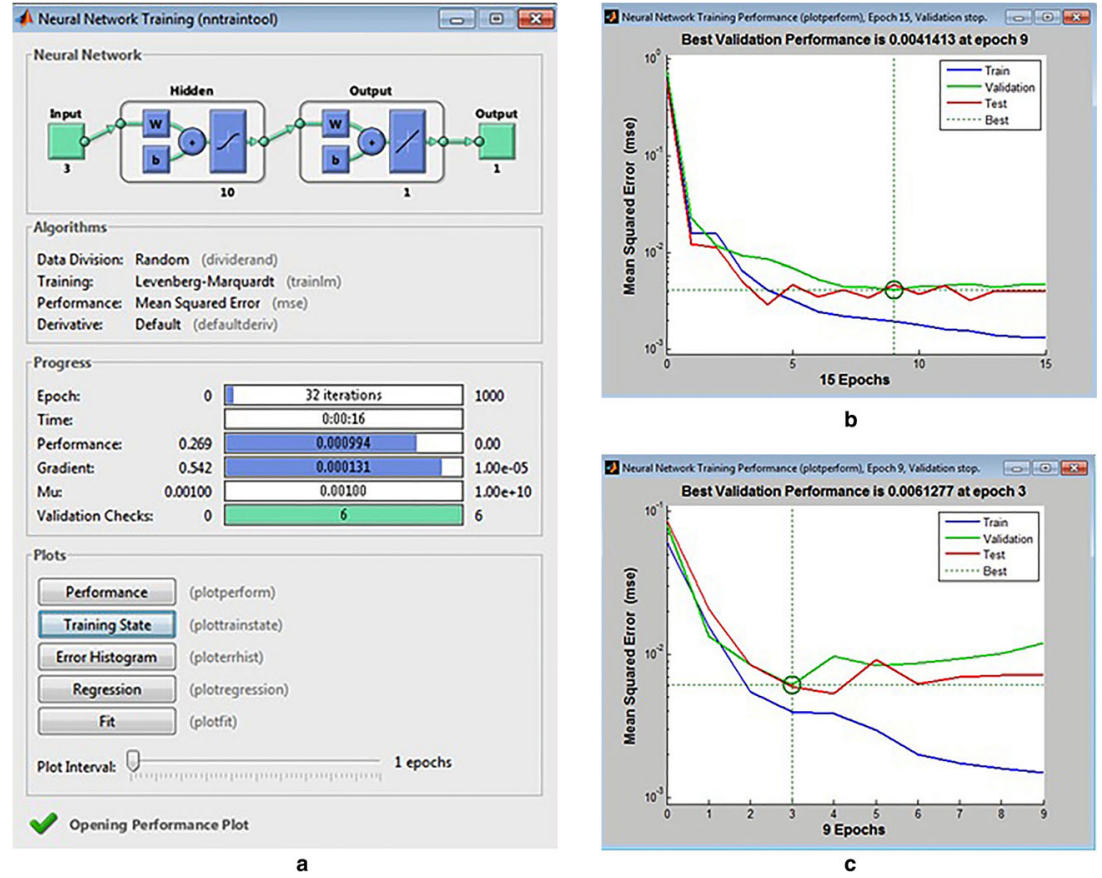

its highly accurate predictions of surface roughness $( \pm$ $5.78 \%)$.

A similar performance appeared to be the best at generalization performance in the cases of allocating 10 and $20 \%$ in the validation set of tabular data (see Fig. 4). The errors of both the first and the second training variants were $0.41 \%$ (see Fig. 4b) and $0.61 \%$ (see Fig. 4c), respectively.

The following tendency was revealed when using an MLP 3-10-1. Increases of 0.1 items in both $n$ and $v_{f}$ increased the $R a^{*}$ values by $0.3028(0.073 \mu \mathrm{m})$ and 0.291 items $(0.071 \mu \mathrm{m})$, respectively. An inverse effect of $a_{p}$ on surface roughness was observed: an increase of 0.1 items in $a_{p}$ led to a decrease in $R a^{*}$ of $0.009(0.002 \mu \mathrm{m})$. The weight ratio of the effects of technological parameters on $R a^{*}$ was $a_{p}: n: v_{f}=$ $1: 25: 25$.

\subsection{3. Determining the Pareto curves (the third step of the strategy).}

$R a^{*}$ values, calculated for the experimental values $x_{1}, x_{2}, x_{3}$ (see Tables 3, 4, 5, 6, and 7) using the neural network, are used to show the Pareto frontiers in Fig. 5.
Fig. 5 Pareto frontiers for machining time of unit volume $T_{m}{ }^{*}$ with surface roughness, $R a^{*}$, at a fixed $a_{p}$, and $n$, with varying feed rates $v_{f}$ (at higher feed rates, $R a^{*}$ increases, and $T_{m}{ }^{*}$ decreases)

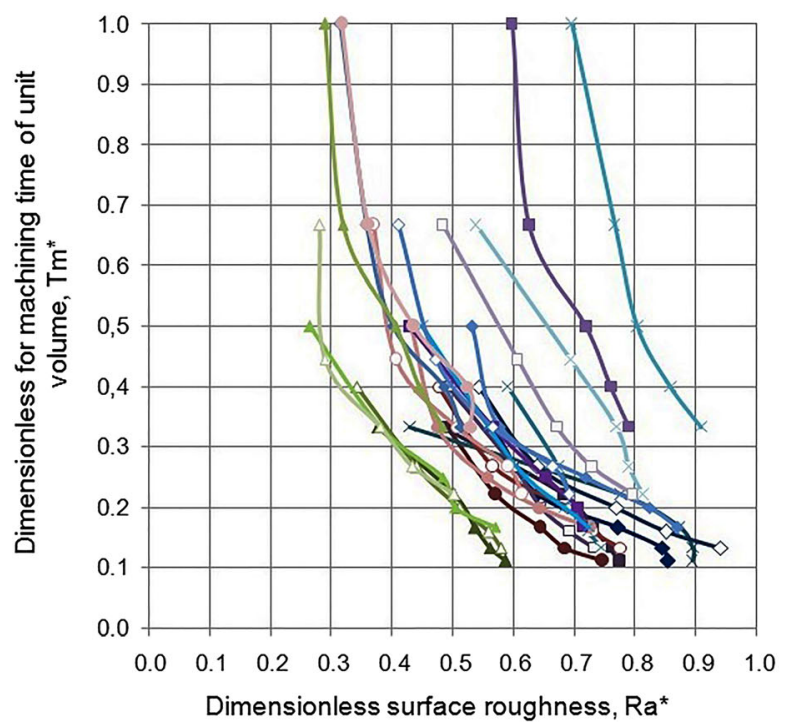




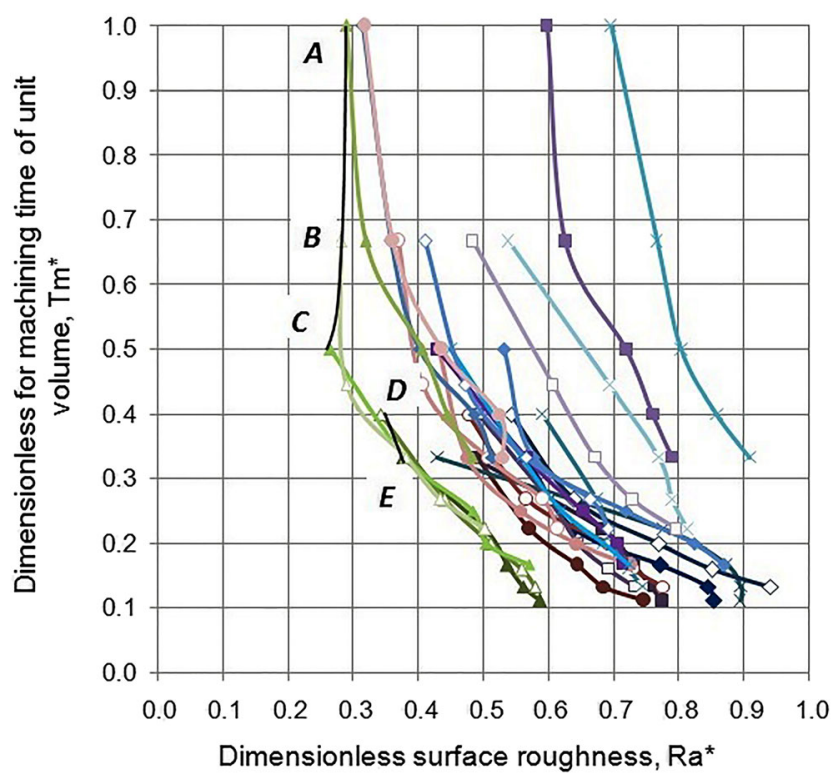

Fig. 6 Intercepts demonstrating the relation of $R a^{*}$ and $T_{m}{ }^{*}: \mathrm{AB}$ corresponds to $n=600 \mathrm{rpm}, v_{f}=50 \mathrm{~mm} / \mathrm{min}, a_{p}=0.5 \ldots 0.75 \mathrm{~mm}$; BC corresponds to $n=600 \mathrm{rpm}, v_{f}=50 \mathrm{~mm} / \mathrm{min}, a_{p}=0.75 \ldots 1.0 \mathrm{~mm}$; DE corresponds to $n=600 \mathrm{rpm}, v_{f}=50 \mathrm{~mm} / \mathrm{min}, a_{p}=1.25 \ldots 1.5 \mathrm{~mm}$. Point coordinates: A (1.000; 0.293), B (0.280;0.666), C (0.262;0.500), D $(0.340 ; 0.400), \mathrm{E}(0.361 ; 0.325)$

Intercepts $\mathrm{AB}, \mathrm{BC}$, and $\mathrm{DE}$ are plotted at the end points of the curves closest to the reference points $R a^{*}$ and $T_{m}{ }^{*}$ (Fig. 6).

Intercept $\mathrm{AB}$ corresponds to $n=600 \mathrm{rpm}, v_{f}=50 \mathrm{~mm} / \mathrm{min}, a$ ${ }_{p}=0.5 \ldots 0.75 \mathrm{~mm}$. Intercept BC corresponds to $n=600 \mathrm{rpm}, v_{f}=$ $50 \mathrm{~mm} / \mathrm{min}, a_{p}=0.75 \ldots 1.0 \mathrm{~mm}$. Intercept $\mathrm{DE}$ corresponds to

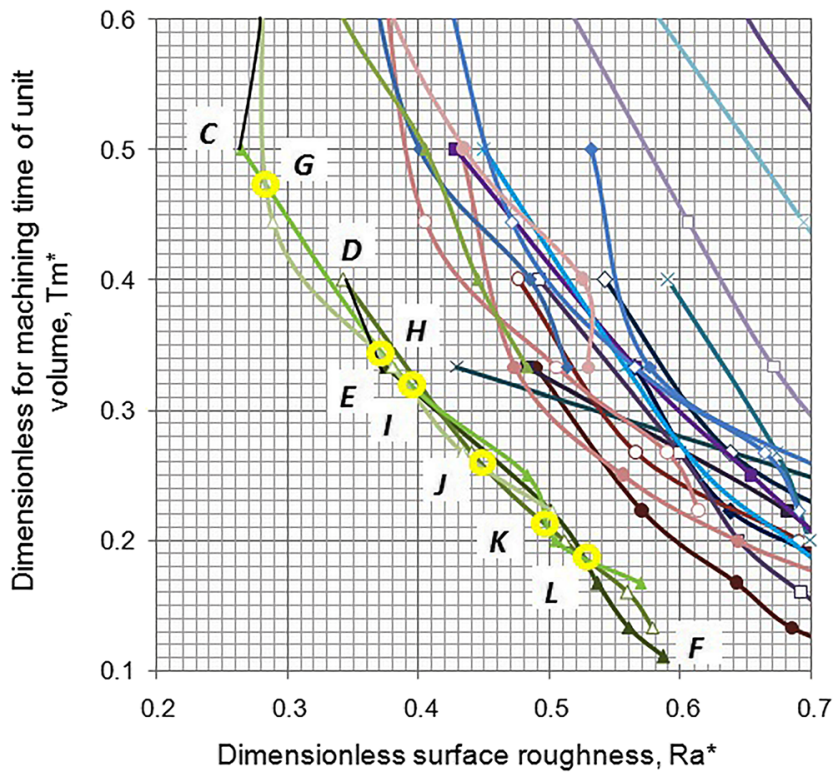

Fig. 7 The intersection coordinates of the plotted intercepts, the $R a^{*}$ and $T_{m}{ }^{*}$ curves closest to the reference points, and the coordinates of their intersections with each other. It includes the lowest point of those functions, crucial for building the Pareto frontier, with the following values: $\mathrm{G}(0.283 ; 0.471), \mathrm{H}(0.360 ; 0.352), \mathrm{I}(0.400 ; 0.310), \mathrm{J}(0.450$; $0.252), \mathrm{K}(0.500 ; 0.215), \mathrm{L}(0.525 ; 0.197)$, and F $(0.582 ; 0.111)$ $n=600 \mathrm{rpm}, v_{f}=50 \mathrm{~mm} / \mathrm{min}, a_{p}=1.25 \ldots 1.5 \mathrm{~mm}$. The coordinates of the intercept ends are $\mathrm{A}(1.000 ; 0.293), \mathrm{B}(0.280 ; 0.666)$, C (0.262; 0.500), D (0.340; 0.400), E (0.361; 0.325). Following the trend of the target function $\mathrm{f}, 11$ points on the Pareto front were established with the following new numbers of the points: $\mathrm{A} \rightarrow$ number $1, \mathrm{~B} \rightarrow$ number $2, \mathrm{C} \rightarrow$ number $3, \mathrm{G} \rightarrow$ number $4, \mathrm{H} \rightarrow$ number $5, \mathrm{E} \rightarrow$ number $6, \mathrm{I} \rightarrow$ number $7, \mathrm{~J} \rightarrow$ number $8, \mathrm{~K} \rightarrow$ number $9, \mathrm{~L} \rightarrow$ number $10, \mathrm{~F} \rightarrow$ number 11 (Fig. 7).

Ten sections were established from the Pareto frontier analysis. Section I, between point 1 and point 2, relates to $a_{p}=0.5 \ldots$ $0.75 \mathrm{~mm}, v_{f}=50 \mathrm{~mm} / \mathrm{min}$, and $n=600 \mathrm{rpm}$; section II between point 2 and point 3 , relates to $a_{p}=0.75 \ldots 1.0 \mathrm{~mm}, v_{f}=50 \mathrm{~mm} /$ min, and $n=600 \mathrm{rpm}$; section III, between point 3 and point 4 , relates to $a_{p}=1.0 \ldots 1.12 \mathrm{~mm}, n=600 \mathrm{rpm}$, and $v_{f}=50 \mathrm{~mm} / \mathrm{min}$; section IV, between point 4 and point 5 , relates to $a_{p}=1.12 \ldots$ $1.25 \mathrm{~mm}, n=600 \mathrm{rpm}$, and $v_{f}=50 \mathrm{~mm} / \mathrm{min}$; section V, between point 5 and point 6 , relates to $a_{p}=1.25 \ldots 1.5 \mathrm{~mm}, v_{f}=50 \mathrm{~mm} /$ min, and $n=600 \mathrm{rpm}$; section VI, between point 6 and point 7 , relates to $a_{p}=1.5 \mathrm{~mm}, n=600 \mathrm{rpm}$, and $v_{f}=50 \ldots 100 \mathrm{~mm} / \mathrm{min}$; section VII, between point 7 and point 8 , relates to $a_{p}=1.5 \ldots$ $0.75 \mathrm{~mm}, n=600 \mathrm{rpm}, v_{f}=100 \mathrm{~mm} / \mathrm{min}$; section VIII, between point 8 and point 9 , relates to $a_{p}=0.75 \ldots 1.25 \mathrm{~mm}, n=600 \mathrm{rpm}$ and $v_{f}=100 \ldots 200 \mathrm{~mm} / \mathrm{min}$; section IX, between point 9 and point 10 , relates to $a_{p}=1.25 \ldots 1.0 \mathrm{~mm}, n=600 \mathrm{rpm}$, and $v_{f=}$ $120 . .150 \mathrm{~mm} / \mathrm{min}$; section $\mathrm{X}$, between point 10 and point 11 , relates to $a_{p}=1.0 \ldots 1.5 \mathrm{~mm}, n=600 \mathrm{rpm}$, and $v_{f}=150 \mathrm{~mm} / \mathrm{min}$. Point 10, at a special point on the Pareto frontier, was at an intersection of three curves for $a_{p}=1.0 \mathrm{~mm}, n=600 \mathrm{rpm}$, and $v_{f}=150 \mathrm{~mm} / \mathrm{min} ; a_{p}=1.25 \mathrm{~mm}, n=600 \mathrm{rpm}$, and $v_{f}=150 \mathrm{~mm} /$ $\min ; a_{p}=1.5 \mathrm{~mm}, n=600 \mathrm{rpm}$, and $v_{f}=150 \mathrm{~mm} / \mathrm{min}$.

\subsection{4. Determining the Pareto frontier (the fourth step of the strategy).}

Expert assessments were employed, so as to narrow the set of Pareto points, in order to determine the higher weighing factor of the machining time criteria, $T_{m}{ }^{*}$, over the surface roughness, $R a^{*}$. As we can see in Fig. 8a, blue line indicates the Pareto non-dominated estimates. The end point of this vector with the coordinates $(0.358 ; 0.358)$. As seen in the figure, point no. 12 can be considered as the non-dominated Pareto point for an unconstrained optimization with equivalent criteria $f_{1}$ and $f_{2}$. The global optima in this case corresponds to the following values: $T_{m}=0.358 \mathrm{~min} / \mathrm{cm} 3, R a=0.087 \mu \mathrm{m}$, $n=600 \mathrm{rpm}, a_{p}=0.75 \mathrm{~mm}$, and $v_{f}=82 \mathrm{~mm} / \mathrm{min}$.

\subsection{Determining the optimal cutting parameters (the fifth step of the strategy)}

In the final step of the algorithm, information gained in the DM was used to set the maximum degradation in the surface roughness value. It was equal to $R a=0.142 \mu \mathrm{m}$ corresponding to the 11th point of the Pareto frontier $(0.582,0.111)-F i g$. $8 \mathrm{~b}$. In this 
Fig. 8 Global optima Pareto frontier for the dimensionless parameters: $T_{m}=0.358 \mathrm{~min} / \mathrm{cm}^{3}$, $R a=0.087 \mu \mathrm{m}, n=600 \mathrm{rpm}$, $a_{p}=0.75 \mathrm{~mm}, v_{f}=82 \mathrm{~mm} / \mathrm{min}$, with the corresponding real parameters: $T_{m}=0.111 \mathrm{~min} / \mathrm{cm}^{3}$, $R a=0.142 \mu \mathrm{m}, n=600 \mathrm{rpm}$, $a_{p}=1.5 \mathrm{~mm}, v_{f}=150 \mathrm{~mm} / \mathrm{min}$

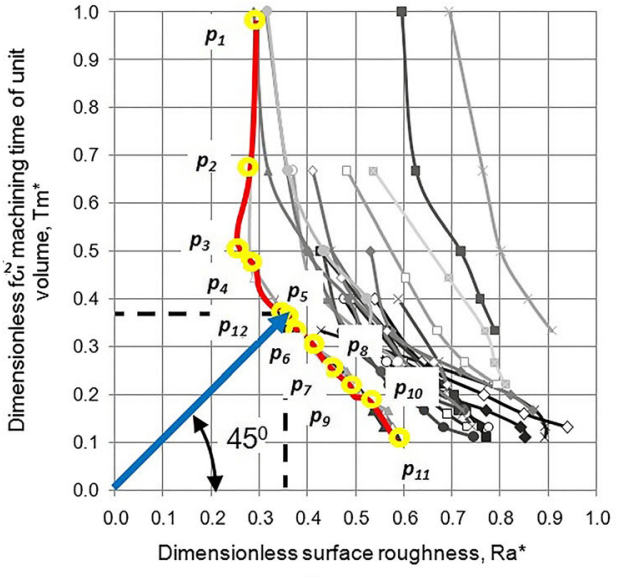

a

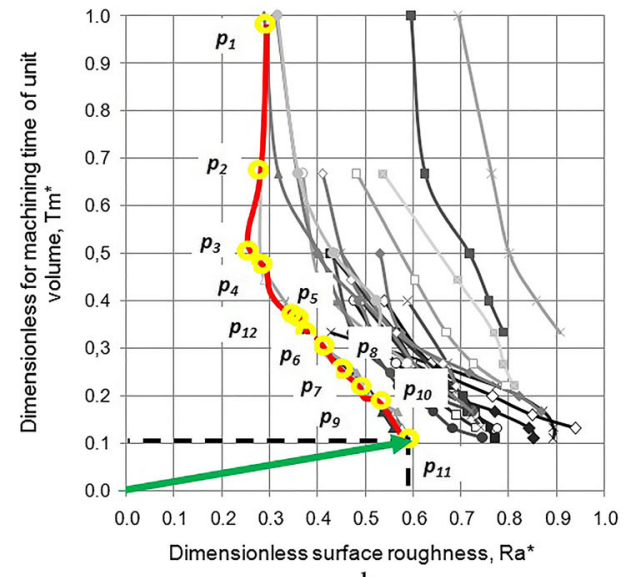

b case, the optimization criteria $T_{m}{ }^{*}$ was five times smaller than $R a$ and for points 12 and 11, the valid preference was $\mathrm{y}_{11} \succ Y \mathrm{y}_{12}$ and the induced preference was $\mathrm{x}_{11} \Leftarrow X \mathrm{x}_{12}$. As a result, the selected points are presented above, the green vector pointing to values of $(0.142,0.111)$, of the optimum cutting parameters $\left(n=600 \mathrm{rpm}, a_{p}=1.5 \mathrm{~mm}, v_{f}=150 \mathrm{~mm} / \mathrm{min}\right.$ ).

Figure $9 \mathrm{a}$ and $\mathrm{b}$ show the profile of the machined surface corresponding to the global optimum conditions $\left(T_{m}=0.358 \mathrm{~min} / \mathrm{cm}^{3}, R a=0.087 \mu \mathrm{m}, n=600 \mathrm{rpm}, a_{p}=\right.$
$0.75 \mathrm{~mm}, v_{f}=82 \mathrm{~mm} / \mathrm{min}$ ), and Fig. 9c, $\mathrm{d}$ shows the same results for the local optimum $\left(T_{m}=0.111 \mathrm{~min} / \mathrm{cm}^{3}, R a=\right.$ $0.142 \mu \mathrm{m}, n=600 \mathrm{rpm}, a_{p}=1.5 \mathrm{~mm}, v_{f}=150 \mathrm{~mm} / \mathrm{min}$ ). The stylus of surface roughness tester moved in a parallel direction to the cutting direction.

In summary, the accurate values of the Pareto curve and the vector coordinates were automatically obtained in Matlab using a customized $\mathrm{NN}$ algorithm. The vector of estimates, $f$, was selected as the optimization criterion, with a boundary

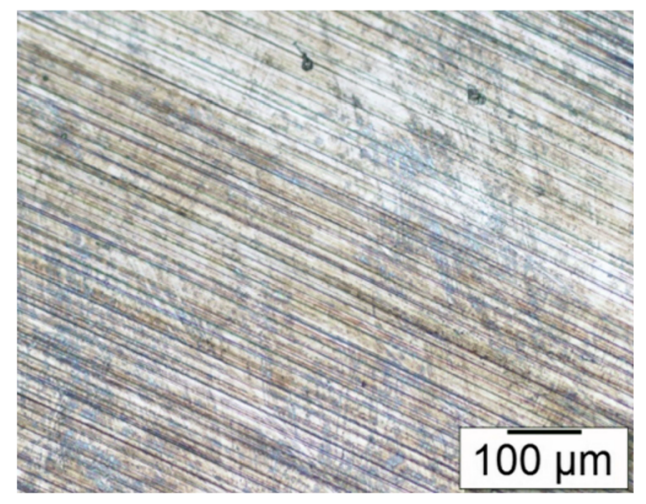

a

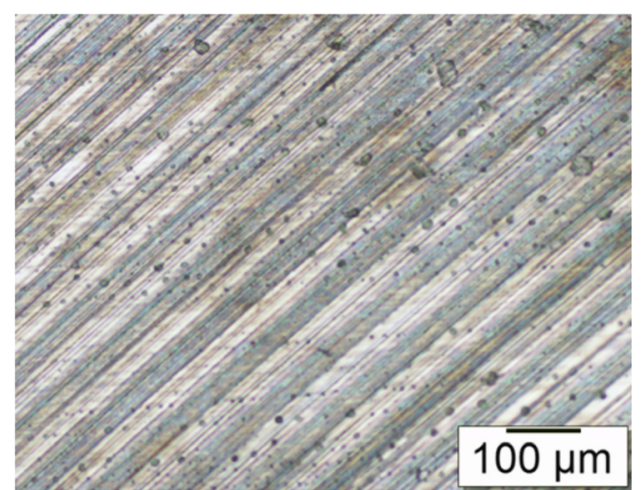

$\mathrm{c}$

Fig. 9 Optical microscopy results and surface profile for the optimized machining conditions. a, b Global optimum $\left(T_{m}=0.358 \mathrm{~min} / \mathrm{cm}^{3}, R a=\right.$ $\left.0.087 \mu \mathrm{m}, n=600 \mathrm{rpm}, a_{p}=0.75 \mathrm{~mm}, v_{f}=82 \mathrm{~mm} / \mathrm{min}\right) . \mathbf{c}, \mathbf{d}$ Local

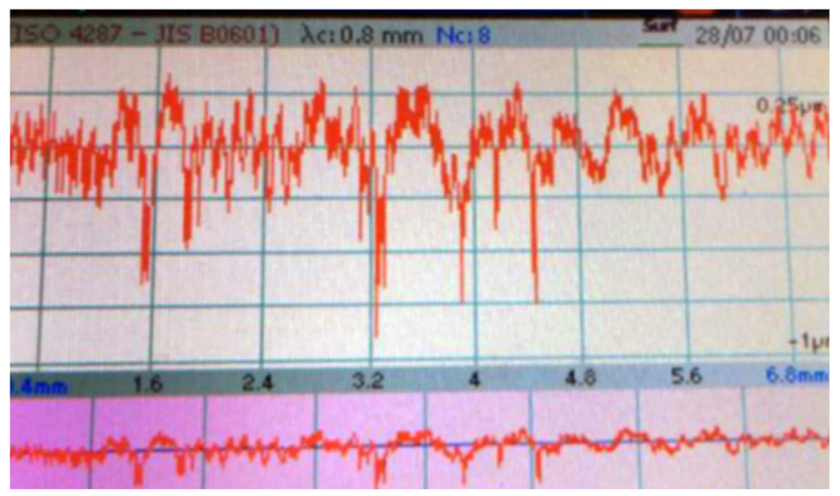

b

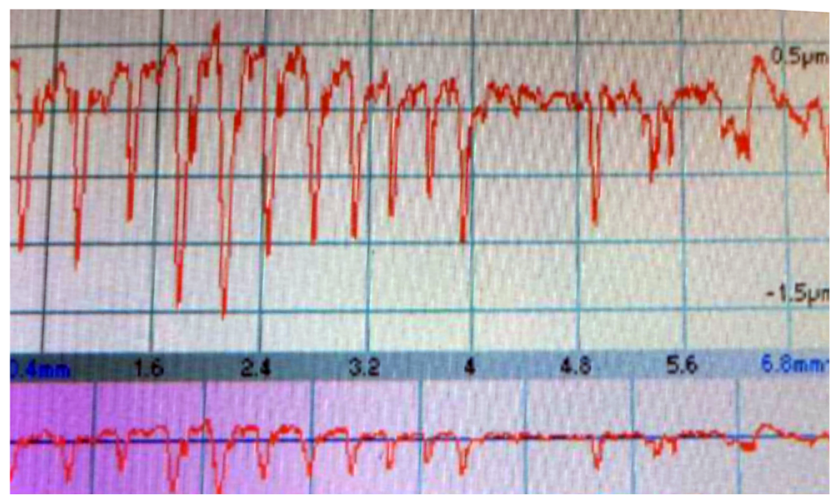

d

optimum $\left(T_{m}=0.111 \mathrm{~min} / \mathrm{cm}^{3}, R a=0.142 \mu \mathrm{m}, n=600 \mathrm{rpm}, a_{p}=\right.$ $\left.1.5 \mathrm{~mm}, v_{f}=150 \mathrm{~mm} / \mathrm{min}\right)$ 
limit of $T_{m}{ }^{*} / R a^{*}=1 / 5$. The Newton method with quadratic convergence was employed to solve the non-linear constrained optimization problem. The algorithm implemented in Matlab permitted rapid convergence towards the local optimal cutting parameters in $\mathrm{CNC}$ robot-aided machining depending on spindle speed and depth and their limitations.

The Edgeworth-Pareto method in the analyses generated the optimal surface treatment conditions for highstrength grade- $\mathrm{H}$ steel as part of the experimental conditions. In the analyses that were performed, as well as the variable cutting depth values, the machine tool settings were used as input parameters: $n$-spindle speed, $v_{f}$ feed table.

Experiments were performed using a 5-tooth cutter with a diameter of $63 \mathrm{~mm}$. The results could not therefore be used for other parameters of the tool and machine tool settings. Further analysis was performed to apply the other analytical results under other conditions (tool diameter, number of teeth) relating to high-strength grade-H steel processing.

The cutting speeds corresponding to the test conditions were determined (Table 8).

The milling process was significantly influenced by feed rate per tooth. The analyses were performed at the table feed, $v_{f}$

Based on the known dependence, the feed rate per tooth values were determined (Table 9) for the different machining process conditions used in the tests $\left(n\right.$ and $\left.v_{f}\right)$, as presented in Table 9 and Fig. 10.

There was no reduction in surface roughness at low feed rate values per tooth, $f_{z}$. One reason was the minimal thickness of the uncut chip [45-49]. The influence of the feed rate on surface condition was not observable in the curves of the graphs (Figs. 5, 6, and 7).

The cutting speed in the tests and the feed-rate values per tooth can be adapted to incorporate the test results and analyses from other steel processing conditions.

On the basis of the analysis of the optimal conditions for the tool $(R a=0.142 \mu \mathrm{m}$ at a minimal unit-volume machining time of $T_{m}=0.111 \mathrm{~min} / \mathrm{cm}^{3}$ ) that has been presented, the following machining values were obtained: $v_{c}=118.69 \mathrm{~m} / \mathrm{min}$ and $f_{z}=0.05 \mathrm{~mm} /$ tooth at $a_{p}=1.5 \mathrm{~mm}$. These values can be used for other cutters and machine tools in the milling of highstrength grade- $\mathrm{H}$ steel.

Table 8 Cutting speed for different spindle speed values $(\mathrm{d}=63 \mathrm{~mm})$

\begin{tabular}{lcclll}
\hline Spindle speed, $\mathrm{n},(\mathrm{rpm})$ & 400 & 500 & 600 & 700 & 800 \\
Cutting speed, $\mathrm{v}_{\mathrm{c}},(\mathrm{m} / \mathrm{min})$ & 79.13 & 98.91 & 118.69 & 138.47 & 158.26 \\
\hline
\end{tabular}

Table 9 Values of feed per tooth, $f_{z}$, for different relations of parameters $n$ and $v_{f}$ used in the experiment

\begin{tabular}{lrlllll}
\hline & \multicolumn{6}{c}{ Spindle speed, $n,(\mathrm{rpm})$} \\
\cline { 2 - 7 } & & 400 & 500 & 600 & 700 & 800 \\
\hline Feed rate, $v_{f},(\mathrm{~mm} / \mathrm{min})$ & 50 & 0.025 & 0.020 & 0.017 & 0.014 & 0.013 \\
& 75 & 0.038 & 0.030 & 0.025 & 0.021 & 0.019 \\
& 100 & 0.050 & 0.040 & 0.033 & 0.029 & 0.025 \\
& 125 & 0.063 & 0.050 & 0.042 & 0.036 & 0.031 \\
& 150 & 0.075 & 0.060 & 0.050 & 0.043 & 0.038 \\
\hline
\end{tabular}

\subsection{Estimating the cost and power of milling operations for the Pareto curve}

The cost price of processing one part, $C_{i}$, is determined by the formula:

$C_{i}=\left(C_{M h} \times T^{\prime}\right)+\left(C_{\text {Toolmin }} \times T^{\prime}\right)+C_{w}$,

where turning time is $T^{\prime}=\left(L+l_{1}\right) /\left(n \times v_{f}\right)$, where $n=(1000 \times$ $\left.v_{c}\right) /\left(\pi \times d_{t}\right)$; (4) where $C_{M h}-$ cost of machining per hour (SR 400) $\left(C_{M h}=\$ 140\right) ; C_{\text {Toolh }}$-cost of tool holder $\left(C_{\text {Toolh }}=\$ 100\right) ; L_{\text {TToolh }}-$ tool holder life $\left(L_{\text {TToolh }}=5\right.$ Year $\times$ 365 Day $\times 24 \mathrm{~h}=31,536,000 \mathrm{~min}) ; C_{I n}$-cost of insert $\left(C_{I n}=\$ 14\right) ; k$-insert setup $(k=4) ; z$-number of cutting teeth (edges) $(z=5) ; C_{w}$-unit Cost of workpiece $\left(C_{w}=\$ 23\right) ; T$-tool life $(T=60 \mathrm{~min}) ; C_{\text {Toolmin }}$-tool cost per minute $\left(C_{\text {Toolmin }}=\left[\left(C_{I n} \times z\right) /\left(T \times k^{\prime}\right)\right]+\left(C_{\text {Toolh }} \times\right.\right.$ $\left.L_{\text {TToolh }}\right)=\$ 0.33$ ).

The tool manufacturer, Sandvik, offers a formula for the calculation of cutting power (cutting power) $(\mathrm{kW})$ :

$P_{c}=\left(a_{p} \times a_{e} \times v_{f} \times K_{c}\right) /(60 \times 106)$

where $a_{e}$-work engagement (milling width) $\left(a_{e}=40 \mathrm{~mm}\right.$ in our case); $K_{c}$ - specific power (specific energy) N/mm ${ }^{2}\left(K_{c}=\right.$ $3750 \mathrm{~N} / \mathrm{mm}^{2}$ for high-strength steel grade-H).

If we calculate the cost of the milling operation and the input cutting power along the Pareto curve from point 1 to point 11 (see Fig. 8) in accordance with formulas (4) and (5), then $C$ appears to decrease from $\$ 27.08$ to $\$ 24.36$, and $P_{c}$ increases from $0.0625 \mathrm{~kW}$ to $0.5625 \mathrm{~kW}$. The values of these parameters for the optimums are global optimum $C=\$ 25.48, P_{c}=0.359 \mathrm{~kW}$, local optimum $C=\$ 24.36, P_{c}=0.5625 \mathrm{~kW}$. The milling conditions change from the blue vector to the green one at the same time as $T_{m}$ increases 3.2 times and $P_{c} 1.5$ times, while the manufacturing cost decreases by $5 \%$. 
Fig. 10 Results of theoretical $f_{z}$ values used in the experiments presented in the paper

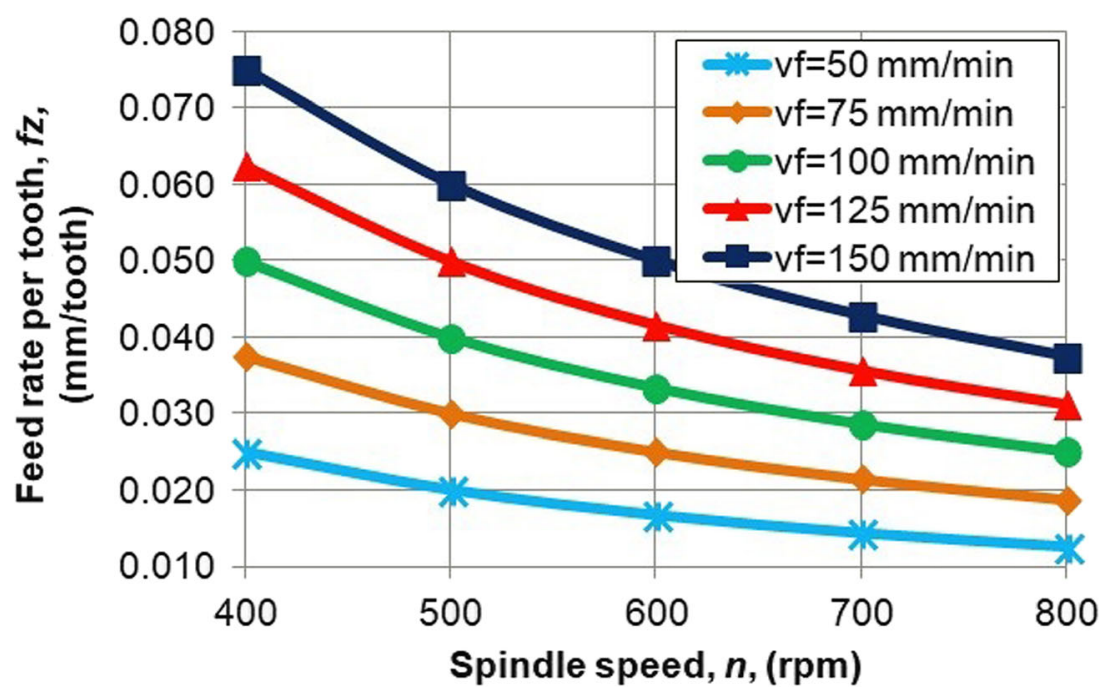

The tests in the form of the global and local optima, obtained by analyzing the test results with the methodology based on the use of an ANN together with Edgeworth-Pareto methods, can be applied to other technological conditions for Grade-H steel face-milling machining, by adjusting the cutting speed, $v_{c}$; the feed per tooth, $f_{z}$; and the cutting inserts.

Funding information The Deanship of Scientific Research at King Saud University funded this work through research group no. (RGP-1439020). The research was also supported through Act 211 Government of the Russian Federation, contract Nr 02.A03.21.0011.

Open Access This article is distributed under the terms of the Creative Commons Attribution 4.0 International License (http:// creativecommons.org/licenses/by/4.0/), which permits unrestricted use, distribution, and reproduction in any medium, provided you give appropriate credit to the original author(s) and the source, provide a link to the Creative Commons license, and indicate if changes were made.

\section{References}

1. Pimenov DY, Guzeev VI, Krolczyk G, Mia M, Wojciechowski S (2018) Modeling flatness deviation in face milling considering angular movement of the machine tool system components and tool flank wear. Precis Eng 54:327-337. https://doi.org/10.1016/j. precisioneng.2018.07.001

2. Pimenov DY (2014) Experimental research of face mill wear effect to flat surface roughness. J Frict Wear 35(3):250-254. https://doi. org/10.3103/S1068366614030118

3. Karkalos NE, Galanis NI, Markopoulos AP (2016) Surface roughness prediction for the milling of Ti-6Al-4V ELI alloy with the use of statistical and soft computing techniques. Measurement 90:2535. https://doi.org/10.1016/j.measurement.2016.04.039

4. Wojciechowski S, Twardowski P, Pelic M, Maruda RW, Barrans S, Krolczyk GM (2016) Precision surface characterization for finish cylindrical milling with dynamic tool displacements model. Precis Eng 46:158-165. https://doi.org/10.1016/j.precisioneng.2016.04. 010 pared to the global optimum. 
5. Pimenov DY, Hassui A, Wojciechowski S, Mia M, Magri A, Suyama DI, Bustillo A, Krolczyk G, Gupta MK (2019) Effect of the relative position of the face milling tool towards the workpiece on machined surface roughness and milling dynamics. Appl Sci 9(5):842. https://doi.org/10.3390/app9050842

6. Mia M, Bashir MA, Khan MA, Dhar NR (2017) Optimization of MQL flow rate for minimum cutting force and surface roughness in end milling of hardened steel (HRC 40). Int J Adv Manuf Technol 89(1-4):675-690. https://doi.org/10.1007/s00170-016-9080-8

7. Pimenov DY, Bustillo A, Mikolajczyk T (2018) Artificial intelligence for automatic prediction of required surface roughness by monitoring wear on face mill teeth. J Intell Manuf 29(5):10451061. https://doi.org/10.1007/s10845-017-1381-8

8. Sun L, Gan B, Cui D (2016) Study on the performance of the shape memory alloy as the shear connector of the steel frame concrete wall structure. Shenyang Jianzhu Daxue Xuebao (Ziran Kexue Ban)/Journal of Shenyang Jianzhu University (Nat Sci) 32(3): 411-419. https://doi.org/10.11717/j.issn:2095-1922.2016.03.04

9. Bruni C, d'Apolito L, Forcellese A, Gabrielli F, Simoncini M (2008) Surface roughness modelling in finish face milling under MQL and dry cutting conditions. Int J Mater Form 1(SUPPL.1): 503-506. https://doi.org/10.1007/s12289-008-0151-8

10. Lela B, Bajić D, Jozić S (2009) Regression analysis, support vector machines, and Bayesian neural network approaches to modeling surface roughness in face milling. Int $\mathrm{J}$ Adv Manuf Technol 42(11-12):1082-1088. https://doi.org/10.1007/s00170-008-1678-z

11. Kovac P, Rodic D, Pucovsky V, Savkovic B, Gostimirovic M (2013) Application of fuzzy logic and regression analysis for modeling surface roughness in face milling. J Intell Manuf 24(4): 755-762. https://doi.org/10.1007/s10845-012-0623-z

12. Simunovic G, Simunovic K, Saric T (2013) Modelling and simulation of surface roughness in face milling. Int J Simul Model 12(3): 141-153. https://doi.org/10.2507/IJSIMM12(3)1.219

13. Pimenov DY (2013) Geometric model of height of microroughness on machined surface taking into account wear of face mill teeth. $\mathrm{J}$ Frict Wear 34(4):290-293. https://doi.org/10.3103/ S1068366613040089

14. Werda S, Duchosal A, Le Quilliec G, Morandeau A, Leroy R (2017) Minimum quantity lubrication advantages when applied to insert flank face in milling. Int J Adv Manuf Technol 92(5-8): 2391-2399. https://doi.org/10.1007/s00170-017-0317-y

15. Baek DK, Ko TJ, Kim HS (1997) A dynamic surface roughness model for face milling. Precis Eng 20(3):171-178

16. Benardos PG, Vosniakos GC (2002) Prediction of surface roughness in CNC face milling using neural networks and Taguchi's design of experiments. Robot Comput Integr Manuf 18(5-6):343354. https://doi.org/10.1016/S0736-5845(02)00005-4

17. Yazdi MRS, Chavoshi SZ (2010) Analysis and estimation of state variables in CNC face milling of AL6061. Prod Eng 4(6):535-543. https://doi.org/10.1007/s11740-010-0232-7

18. Rosales A, Vizán A, Diez E, Alanís A (2010) Prediction of surface roughness by registering cutting forces in the face milling process. Eur J Sci Res 41(2):228-237

19. Bajić D, Celent L, Jozić S (2012) Modeling of the influence of cutting parameters on the surface roughness, tool wear and cutting force in face milling in off-line process control. Strojniski Vestn J Mech Eng 58(11):673-682. https://doi.org/10.5545/sv-jme.2012. 456

20. Chavoshi SZ (2013) Modelling of surface roughness in CNC face milling of alloy stellite 6. Int J Comput Mater Sci Surf Eng 5(4): 304-321. https://doi.org/10.1504/IJCMSSE.2013.059121

21. Saric T, Simunovic G, Simunovic K (2013) Use of neural networks in prediction and simulation of steel surface roughness. Int J Simul Model 12(4):225-236. https://doi.org/10.2507/IJSIMM12(4)2.241

22. Samtas G (2014) Measurement and evaluation of surface roughness based on optic system using image processing and artificial neural network. Int J Adv Manuf Technol 73(1-4):353-364. https://doi. org/10.1007/s00170-014-5828-1

23. Sheth S, George PM (2016) Experimental investigation and prediction of flatness and surface roughness during face milling operation of WCB material. Procedia Technol 23:344-351. https://doi.org/10. 1016/j.protcy.2016.03.036

24. Simunovic G, Svalina I, Simunovic K, Saric T, Havrlisan S, Vukelic D (2016) Surface roughness assessing based on digital image features. Adv Prod Eng Manag 11(2):93-104. https://doi. org/10.14743/apem2016.2.212

25. Bajić D, Lela B, Živković D (2008) Modeling of machined surface roughness and optimization of cutting parameters in face milling. Metalurgija 47(4):331-334

26. Aykut Ș, Demetgul M, Tansel IN (2010) Selection of optimum cutting condition of cobalt-based superalloy with GONNS. Int J Adv Manuf Technol 46(9-12):957-967. https://doi.org/10.1007/ s00170-009-2165-x

27. Sukumar MS, Venkata Ramaiah P, Nagarjuna A (2014) Optimization and prediction of parameters in face milling of Al6061 using taguchi and ANN approach. Procedia Eng 97:365-371. https://doi.org/10.1016/j.proeng.2014.12.260

28. Simunovic K, Simunovic G, Saric T (2015) Single and multiple goal optimization of structural steel face milling process considering different methods of cooling/lubricating. J Clean Prod 94:321329. https://doi.org/10.1016/j.jclepro.2015.02.015

29. Moghaddam MA, Kolahan F (2016) Application of orthogonal array technique and particle swarm optimization approach in surface roughness modification when face milling AISI1045 steel parts. J Ind Eng Int 12(2):199-209. https://doi.org/10.1007/ s40092-015-0137-3

30. Rodríguez J, Quintana G, Bustillo A, Ciurana J (2017) A decisionmaking tool based on decision trees for roughness prediction in face milling. Int J Comput Integr Manuf 30(9):943-957. https://doi.org/ 10.1080/0951192X.2016.1247991

31. Fratila D, Caizar C Application of Taguchi method to selection of optimal lubrication and cutting conditions in face milling of AlMg3. J Clean Prod 19(6-7):640-645. https://doi.org/10.1016/j.jclepro. 2010.12.007

32. Yang W-A, Guo Y, Liao W (2011) Multi-objective optimization of multi-pass face milling using particle swarm intelligence. Int $\mathrm{J}$ Adv Manuf Technol 56(5-8):429-443. https://doi.org/10.1007/s00170011-3187-8

33. Abbas AT, Ragab AE, Al Bahkali EA, El Danaf EA (2016) Optimizing cutting conditions for minimum surface roughness in face milling of high strength steel using carbide inserts. Adv Mater Sci Eng 7372132:1-14. https://doi.org/10.1155/2016/7372132

34. Feng C-XJ YZ-GS, Kingi U, Pervaiz Baig M (2005) Threefold vs. fivefold cross validation in one-hidden-layer and two-hidden-layer predictive neural network modeling of machining surface roughness data. J Manuf Syst 24(2):93-107. https://doi.org/10.1016/ S0278-6125(05)80010-X

35. Feng CX-J, Yu Z-GS, Emanuel JT, Li P-G, Shao X-Y, Wang Z-H (2008) Threefold versus fivefold cross-validation and individual versus average data in predictive regression modelling of machining experimental data. Int J Comput Integr Manuf 21(6):702-714. https://doi.org/10.1080/09511920701530943

36. Asilturk I, Kahramanli H, El Mounayri H (2012) Prediction of cutting forces and surface roughness using artificial neural network (ANN) and support vector regression (SVR) in turning 4140 steel. Mater Sci Technol 28(8):980-986. https://doi.org/10.1179/ 1743284712Y.0000000043

37. Abbas AT, Pimenov DY, Erdakov IN, Mikolajczyk T, El Danaf EA, Taha MA (2017) Minimization of turning time for high-strength steel with a given surface roughness using the Edgeworth-Pareto optimization method. Int J Adv Manuf Technol 93(5-8):23752392. https://doi.org/10.1007/s00170-017-0678-2 
38. Abbas AT, Pimenov DY, Erdakov IN, Taha MA, El Rayes MM, Soliman MS (2018) Artificial intelligence monitoring of hardening methods and cutting conditions and their effects on surface roughness, performance, and finish turning costs of solid-state recycled aluminum alloy 6061 chips. Metals 8(6):394. https://doi.org/10. 3390/met8060394

39. Abbas AT, Pimenov DY, Erdakov IN, Taha MA, Soliman MS, El Rayes MM (2018) ANN surface roughness optimization of AZ61 magnesium alloy finish turning: minimum machining times at prime machining costs. Materials 11(5):808. https://doi.org/10. 3390/ma11050808

40. Iqbal A, He N, Li L, Dar NU (2017) A fuzzy expert system for optimizing parameters and predicting performance measures in hard-milling process. Expert Syst Appl 32(4):1020-1027. https:// doi.org/10.1016/j.eswa.2006.02.003

41. Choudhary AK, Harding JA, Tiwari MK (2009) Data mining in manufacturing: a review based on the kind of knowledge. J Intell Manuf 20(5):501-521. https://doi.org/10.1007/s10845-008-0145-x

42. Kurra S, Hifzur Rahman N, Regalla SP, Gupta AK (2015) Modeling and optimization of surface roughness in single point incremental forming process. J Mater Res Technol 4(3):304-313. https://doi.org/10.1016/j.jmrt.2015.01.003

43. Torabi AJ, Er MJ, Li X, Lim BS, Peen GO (2016) Application of clustering methods for online tool condition monitoring and fault diagnosis in high-speed milling processes. IEEE Syst J 10(2): 7107977:721-7107977:732. https://doi.org/10.1109/JSYST.2015. 2425793
44. Nogin VD (2002) Decision making in multicriteria environment: quantitative approach. M.: FIZMATLIT, p 144. [in Russian]

45. Liu X, DeVor RE, Kapoor SG (2006) An analytical model for the prediction of minimum chip thickness in micromachining. J Manuf Sci Eng 128(2):474-481. https://doi.org/10.1115/1.2162905

46. Liu Z, Shi Z, Wan Y (2013) Definition and determination of the minimum uncut chip thickness of microcutting. Int $J$ Adv Manuf Technol 69(5-8):1219-1232. https://doi.org/10.1007/s00170-0135109-4

47. Mikolajczyk T (2014) Modeling of minimal thickness cutting layer influence on surface roughness in turning. Appl Mech Mater 656: 262-269. https://doi.org/10.4028/www.scientific.net/AMM.656. 262

48. Oliveira FB, Rodriges AR, Coelho RT, Souza AF (2015) Size effect and minimum chip thickness in micromilling. Int $\mathrm{J}$ Mach Tools Manuf 89:39-54. https://doi.org/10.1016/j.ijmachtools.2014.11. 001

49. Rezaei H, Sadeghi MH, Budak E (2018) Determination of minimum uncut chip thickness under various machining conditions during micro-milling of Ti-6Al-4V. Int J Adv Manuf Technol 95(5-8): 1617-1634. https://doi.org/10.1007/s00170-017-1329-3

Publisher's note Springer Nature remains neutral with regard to jurisdictional claims in published maps and institutional affiliations. 